\title{
Les économistes universitaires français et leurs homologues anglais et américains : aspects institutionnels, culturels et linguistiques
}

Jacqueline Percebois

\section{OpenEdition}

Journals

Édition électronique

URL : http://journals.openedition.org/asp/709

DOI : 10.4000/asp.709

ISBN : 978-2-8218-0402-9

ISSN : 2108-6354

\section{Éditeur}

Groupe d'étude et de recherche en anglais de spécialité

\section{Édition imprimée}

Date de publication : 1 décembre 2006

Pagination : 79-91

ISSN : 1246-8185

\section{Référence électronique}

Jacqueline Percebois, "Les économistes universitaires français et leurs homologues anglais et américains : aspects institutionnels, culturels et linguistiques », ASp [En ligne], 49-50 | 2006, mis en ligne le 08 février 2010, consulté le 22 mars 2021. URL : http://journals.openedition.org/asp/709 ; DOI : https://doi.org/10.4000/asp.709

Ce document a été généré automatiquement le 22 mars 2021.

Tous droits réservés 


\title{
Les économistes universitaires
} français et leurs homologues anglais et américains : aspects institutionnels, culturels et linguistiques

\author{
Jacqueline Percebois
}

1 Cette recherche s'inscrit au sein d'un domaine particulier de l'anglais de spécialité ou English for Specific Purposes (ESP) : English for Economics. Elle se situe également, de façon spécifique, dans un contexte d'étude et d'emploi de l'anglais académique ou universitaire, English for Academic purposes (EAP), c'est-à-dire

Language research and instruction that focuses on the specific communicative needs and practices of particular groups in academic contexts. It means grounding instruction in an understanding of the cognitive, social and linguistic demands of specific academic disciplines. (Hyland \& Hamp-Lyons $2002: 2$ )

2 Par conséquent, nous dirons que cette étude relève du domaine d'English for Specific Academic Purposes (ESAP), l'anglais de spécialité universitaire. Quelle communauté linguistique cette étude aborde-t-elle?

3 Selon la définition de Dudley-Evans et St John (2001 : xiv), le concept de communauté linguistique implique " a group who in professional contexts communicate with each other and have therefore developed mechanisms for doing so ». Nous nous intéressons ici aux économistes universitaires français et à leurs homologues britanniques et américains.

4 Le développement de la science économique enseignée à l'université se rattache à des processus historiques spécifiques à chaque pays; il dépend des institutions et des cadres nationaux de pensée.

Durant tout le $20^{\mathrm{e}}$ siècle et dans tous les pays, on observe une institutionnalisation de la discipline économique en même temps qu'une professionnalisation du métier d'économiste, institutionnalisation perceptible par la naissance des départements d'économie dans les universités, d'associations professionnelles, création au niveau 
national et international de distinctions spécifiques récompensant des économistes

au même titre que les chercheurs d'autres disciplines. (Lebaron $2000: 18$ ) financement.
findion d'assor

En effet, selon les termes du professeur Alain Touraine (1972:163),

le professeur universitaire est un Janus bifrons: expert, il est tourné vers l'extérieur; chercheur, il est tourné vers l'intérieur de l'université. Sociologiquement il n'est pas suffisant de reconnaître le niveau scientifique et technique élevé d'un enseignement pour parler de sa professionnalisation. Il faut encore que les professeurs forment une association capable de définir un cadre de conduite, en particulier les conditions d'accès dans le groupe et possédant la capacité de sanctionner les manquements aux règles déontologiques ou d'agir pour la défense des intérêts de la collectivité.

Cette autonomie d'organisation, de décision et de jugement sépare les professionnels des techniciens, quel que soit leur niveau.

7 Les aspects traités dans cette première partie sont donc bien des indices de la professionnalisation des academic economists. Dans une deuxième partie, nous aborderons leur discours qui se caractérise aujourd'hui par la prédominance de deux langages : les mathématiques et l'anglais. Quelles sont les raisons de cette évolution ? Pourquoi l'anglais s'impose-t-il comme langue véhiculaire des academic economists, ces professionnels dont les points communs sont l'enseignement de l'économie à l'université et la langue qui leur sert de référence intellectuelle ? Si l'expression en anglais est naturelle pour les britanniques et les américains, pourquoi s'impose-t-elle pour les économistes universitaires français?

\section{Convergences et divergences institutionnelles et culturelles}

Historiquement, "C'est en Angleterre puis aux États-Unis que la représentation des économistes comme profession a pris toute sa force» (Lebaron 2000 : 18). Pour chacun des pays concernés, l'Angleterre, les États-Unis et la France, nous allons étudier les circonstances de la création des enseignements, la fondation d'associations, de revues et de récompenses ainsi que les modes de recrutement et de financement.

\subsection{Création et évolution des enseignements}

\subsubsection{En Angleterre}

9 À la fin du $19^{e}$ siècle l'enseignement de l'économie n'est qu'une matière subsidiaire des cours d'histoire à Oxford et de science morale à Cambridge. D'une part, les sujets pratiques sont exclus de l'enseignement universitaire ; d'autre part, l'université est destinée à instruire des élites cultivées plutôt qu'à former ou non des professionnels universitaires (Fourcade-Gourinchas 2002).

La London School of Economics and Political Science est créée en 1895. Après des études de mathématiques à Cambridge, Alfred Marshall y est nommé lecturer in moral science; il devra promettre d'exclure la comptabilité de son enseignement pour faire 
admettre ses Tripos en économie. L'économie doit trouver sa légitimation autour de matières classiques comme la philosophie morale et les mathématiques. Ainsi, l'économie va se développer comme une matière théorique, abstraite, renforçant sa légitimité par les mathématiques. De ce fait les économistes britanniques se considèrent comme des scientifiques à part entière.

\subsubsection{Aux États-Unis}

$11 \mathrm{Au} 19^{\mathrm{e}}$ siècle, et depuis la guerre de sécession, certains principes économiques font partie du programme de morale des colleges, alors que de nouveaux établissements d'enseignement supérieur sont créés.

Les bailleurs de fond des nouvelles universités sont ouverts aux nouvelles formes de connaissances que représentent les sciences sociales auxquelles ils attribuent la vertu de susciter une demande de marché mais aussi le but de servir de guide à la société en se substituant à la morale religieuse pour aider à la résolution rationnelle des problèmes sociaux. (Fourcade-Gourinchas 2002)

12 Les économistes universitaires vont devoir rechercher une identité morale, un statut, dans une culture qui n'a jamais accordé beaucoup de prestige aux activités intellectuelles. On constate un mouvement rapide vers la professionnalisation, mouvement animé de diverses motivations.

Le but avoué est la recherche d'une scientificité irréprochable et la satisfaction de demandes sociales précises exprimées par des acteurs externes à l'institution universitaire. Un professionnalisme étroit est encouragé par les bailleurs de fonds tant privés que publics. D'autre part, ne disposant pas d'une véritable fonction publique d'élite, les administrations publiques vont faire appel aux ressources de l'université comme substitut. Enfin, les économistes universitaires apparaîtront comme investis d'une mission d'agents du progrès social.

\subsubsection{En France}

14 Dès le $17^{e}$ siècle, les économistes physiocrates, parfois appelés «la secte des économistes ", participent par leurs travaux à la naissance de la science politique. Mais ce n'est qu'au $19^{\mathrm{e}}$ siècle que les premières chaires d'enseignement de science politique sont créées au Collège de France, dans les écoles d'ingénieurs et à l'École Libre des Sciences Politiques fondée en 1871. L'État crée ensuite des chaires dans les facultés de droit où, à partir de 1877 , l'économie est une matière secondaire dans les cursus juridiques, enseignée par des juristes. L'enseignement se caractérise par une aversion pour la formulation mathématique; c'est un discours qui tient plus de l'art politique que de la science. Les économistes eux-mêmes, pour la plupart, considèrent l'économie politique comme une science morale.

Walras ne peut obtenir un emploi universitaire en France et part en 1870 enseigner en Suisse, à Lausanne, où une chaire d'économie vient d'être crée à la Faculté de Droit.

Le lien institutionnel avec le droit empêchera longtemps l'affirmation d'un programme scientifique disciplinaire en France. "Les études de sciences économiques (qui s'imposent vraiment face à la notion d'économie politique' dans les années 1960) restent longtemps en France des filières optionnelles au sein des cursus de droit " (Lebaron 2000 : 22). L'économie politique restera sous le contrôle des facultés de droit jusqu'après la $2^{\mathrm{e}}$ guerre mondiale, la licence d'économie n'étant créée qu'en 1961. 


\subsection{Les associations, les revues et les récompenses}

\section{reconnue à travers la création d'associations et de revues pour diffuser les} connaissances et asseoir sa légitimité entre ses pairs.

\subsubsection{En Angleterre}

$\mathrm{Au} 19^{\mathrm{e}}$ siècle, les sujets économiques sont abordés dans les revues intellectuelles comme The Quarterly Review ou The Westminster Review. The Economist est créé en 1843, environ 50 ans avant la parution de l'Economic Journal et la création en 1891 de la British Economic Association qui deviendra la Royal Economic Society fondée sur des critères élitistes d'admission.

19 L'intégration des femmes dans la profession des économistes universitaires est très tardive et limitée. Avant d'épouser Marshall en 1877, Marie Paley avait suivi «the informal scheme of Cambridge lectures for women", le caractère informel de ces enseignements témoignant de leur marginalité. The Royal Economic Society's Committee for Women in Economics n'a été créé qu'en novembre 1996; il a pour but de promouvoir le rôle des femmes dans les professions d'économiste en Angleterre. Ses membres appartiennent à l'université, au monde des affaires ou à la fonction publique. Le comité étudie la place des femmes dans le domaine économique afin d'essayer de réduire leur sous-représentation et d'établir des réseaux en s'intéressant particulièrement à celles dont la carrière débute.

\subsubsection{Aux États-Unis}

20

Dans la deuxième moitié du $19^{\mathrm{e}}$ siècle, le domaine de l'économie politique américain est déjà bien davantage professionnalisé que ses équivalents britannique et français. L'American Economic Association et l'American Economic Review créées en 1885 contribuent fortement au sentiment d'intégration des professeurs. Dépourvues de critères élitistes d'admission, comme par exemple la Royal Economic Society en Angleterre, ou de parti pris idéologique, comme la Société d'Économie politique en France, elles se caractérisent par un fonctionnement démocratique et un recrutement étroitement académique (Fourcade-Gourinchas 2002). D'autre part, l'American Association of University Professors a été créée dès 1915.

\subsubsection{En France}

21 La Société d'Économie politique a été fondée en 1846 par le fils de Jean-Baptiste Say. Société savante plutôt qu'organisation professionnelle, elle réunit des économistes libéraux et publie les Annales d'Économie politique et le Journal des Économistes. C'est en opposition avec le modèle mondain et bourgeois de la Société d'Économie politique que Charles Gide crée en 1887 la Revue d'Économie politique (Lebaron 2000 : 29).

Quant à l'Association française de sciences économiques, elle ne sera créée qu'en 1950. «Il a fallu attendre en France environ un siècle pour que la nouvelle dénomination acquière une reconnaissance collective apparemment équivalente » (Lebaron $2000: 22$ ). Tournée vers la production scientifique en sciences économiques, elle veut se donner 
une identité disciplinaire s'appuyant sur le modèle de l'American Economic Association, à l'opposé du modèle mondain et bourgeois de la Société d'Économie politique. Les enseignements d'économie présentent moins les savoirs ou savoir-faire utilisés dans les entreprises que des sujets d'apparence abstraite, en particulier dans les filières les plus théoriques, ce qui est dénoncé par les professionnels (Lebaron 2000 : 23).

\subsubsection{Les récompenses - Le Prix Nobel}

Sur le plan international, le niveau de reconnaissance académique se mesure par les citations dans le Social Science Citation Index (SSCI). On définit les économistes majeurs comme les universitaires étant cités le plus grand nombre de fois selon le SSCI. Ce dernier donne l'ensemble des noms cités par environ 200 publications économiques, ainsi que par plusieurs centaines de publications dans les autres sciences sociales (Frey 2001 : 1). Avec 13 économistes majeurs, alors que le Royaume-Uni en compte 91, la France se classe au deuxième rang de tous les pays européens.

Selon le Who's Who in Economics (Blaug 1999), 62,8 \% des économistes les plus souvent cités travaillent aux France. La fréquence de citation, du nom et des travaux de recherche, se révèle pertinente tant pour la carrière des intéressés que pour les comparaisons entre pays.

En France, la création d'un prix du Nouvel Economiste comme celle du Conseil d'analyse économique sont considérées parfois comme des «exemples d'intrusion des pouvoirs politiques et médiatiques à l'intérieur du champ» (Blaug 1999: 43). Le prix dit Nobel n'a-t-il pas été créé en 1968, pour le $300^{\mathrm{e}}$ anniversaire de la Banque Royale de Suède, un pouvoir économique (Lebaron $2000: 42)$ ?

Toutefois, le «Prix Nobel» de science économique s'inscrit dans un travail de standardisation et de professionnalisation de la discipline économique. Partout dans le monde, sont adoptés les canons scientifiques produits aux États-Unis; les carrières académiques les plus prestigieuses doivent inclure un passage initiatique par les ÉtatsUnis. Les caractéristiques des lauréats du prix de science économique font ainsi apparaitre une américanisation incontestable. Nombre de Prix Nobel sont disposés à répondre à la demande de prises de position sur l'avenir économique et politique du monde. Citons Milton Friedman prophétisant l'échec de l'euro et, en 2000, sept lauréats américains du Prix Nobel de science économique qui sont intervenus dans la campagne présidentielle américaine pour soutenir le candidat républicain, George W. Bush (Lebaron 2002).

\subsection{Recrutement et financement}

Kenneth Boulding est l'auteur d'un texte intitulé «The Skills of the Economist » (Annexe) dans lequel il s'interroge sur la "profession » des économistes, mettant son talent et son humour au service de sa démonstration, en particulier en lui appliquant des concepts économiques. Ainsi, par exemple, les économistes n'établiraient-ils pas de barrières à l'entrée de leur profession et ne défendraient-ils pas un monopole. Peut-on discerner des différences sur ces points en Angleterre, aux France et en France?

Avec le système anglo-saxon et le système français s'oppose une conception du rôle de l'État libéral ou au contraire centralisateur. D'après M. Fourcade-Gourinchas (2002), on 
observe, d'un côté, les pays anglo-saxons centrés sur un modèle académique de production des connaissances et affirmation disciplinaire dure, de l'autre les pays d'Europe continentale qui relèvent plutôt d'un modèle technocratique. En fait, nous noterons aussi des divergences entre l'Angleterre et les États-Unis.

\subsubsection{En Angleterre} Business School dont le parcours est controversé par ses «academic colleagues who tend to model an economist' career so that he or she does research and writes peer-reviewed journal articles and monographies ".

31 Selon un point commun entre l'Angleterre et les États-Unis, les universités font paraître dans la presse spécialisée les annonces de vacance de postes et les candidats proposent directement leurs services.

32 Le financement est assuré par l'État selon un fixed national salary scale négocié par The Association of University Teachers et The University and Colleges Employer Association. Cette échelle de salaires ne concerne pas les salaires des professeurs qui se négocient entre le service du personnel et l'intéressé (Moore 2002).

\subsubsection{Aux États-Unis}

êtats-Unis, à l'Université comme dans les organismes publics, les économistes sont recrutés suivant des procédures plutôt caractéristiques, en Europe, des entreprises privées (Lebaron $2000: 27$ ). Le PhD est nécessaire pour enseigner à l'université et il existe un marché des «PhD economists ».

Il n'existe pas de ministère de l'éducation nationale au niveau fédéral. Dans chaque État de l'union, le système universitaire public comprend en général plusieurs campus ; son niveau académique dépend de la situation financière de l'État. En effet, chacun des États est libre de décider de sa politique en matière d'enseignement supérieur. Les niveaux sont variables, même si Jacques Attali écrit que certaines des universités publiques peuvent être comparées aux meilleures universités privées, telles les universités de l'Indiana, du Wisconsin, de Californie, qui peuvent être comparées aux plus réputées des universités privées, telles Harvard, Princeton, Stanford ou le MIT (1998: 113).

Chaque université, privée ou publique, se charge du recrutement de ses professeurs. Pour postuler, chaque candidat à un poste d'enseignant doit s'adresser lui-même aux universités qui ont fait paraître dans la presse spécialisée une annonce de vacance de poste correspondant à ses qualifications. Si sa candidature a été retenue par un comité de sélection composé de quelques professeurs du département, le candidat sera reçu par les professeurs et l'administration de l'université. En fonction de leurs qualifications, les candidats se verront offrir divers contrats.

36 Aux États-Unis, le système d'enseignement est d'avantage orienté par des demandes de production ou des exigences du marché du travail (Touraine 1972:122) que par des 
idées sur l'éducation ; la production universitaire est de plus en plus sollicitée par les demandes en provenance des milieux commerciaux et politiques (Fourcade-Gourinchas 2002).

Le financement diffère en fonction du type d'université. Outre des droits de scolarité élevés perçus par tous les types d'établissement, les universités privées sont financées par des dons privés tandis que les universités publiques sont financées par des subventions d'État (Moore 2002). Les fonds fédéraux financent surtout les sciences naturelles et médicales. Les sciences sociales reçoivent l'essentiel de leurs fonds de recherche de fondations comme la fondation Ford.

Le contrôle professionnel des carrières s'organise à l'intérieur des départements. La règle tacite $\mathrm{du}$ « up or out » (promotion ou expulsion) s'applique à ceux qui n'ont pas la sécurité de l'emploi, grâce à une nomination permanente (tenure) (Touraine 1972 : 164). En définitive, la carrière d'un enseignant ne tient pas à la qualité de son enseignement mais à sa production scientifique, ce qui s'exprime par publish or perish.

\subsubsection{En France}

En France, le doctorat d'économie conditionne l'occupation d'un poste universitaire au sein de la $5^{\mathrm{e}}$ Section du Conseil National des Universités (CNU), après passage par un concours national.

40 Au sommet de la hiérarchie, les économistes ont souvent complété leur formation initiale aux États-Unis. Nous citerons des économistes toulousains liés au MIT : Jean Tirole, Jean-Jacques Laffont, qui occupent une position très élevée du point de vue des classements internationaux (Lebaron $2000: 56$ ).

41 Étant en grande partie un bien public, l'enseignement supérieur français fait l'objet de choix budgétaires soumis à l'appréciation du Parlement (Bienaymé 1986: 72). Les ressources publiques alimentent les activités universitaires par le canal de l'État (95\%), ou par les collectivités locales.

\section{Le discours des academic economists}

Dans l'avant-propos du Dictionnaire PUF de Sciences économiques (2001), les professeurs Gaumont, Jessua, Labrousse et Vitry écrivent que l'économie est une discipline «si vulnérable aux partis pris idéologiques et si imbriquée dans les affaires du siècle qu'on lui a souvent dénié le caractère de science ». Dans la préface, Gérard Debreu (Prix Nobel 1983) souligne le chemin parcouru depuis la publication du Dictionary of Political Economy publié par Palgrave en 1894. Le nom de la discipline lui-même a changé, « science économique » ayant remplacé « économie politique ». Nous avons également noté les multiples facteurs qui ont influé sur cette science au cours du temps et qui sont susceptibles d'interagir à un moment donné.

À partir de cette diversité, peut-on dégager quelques traits fondamentaux du discours des économistes universitaires? Un constat s'impose d'abord: la domination américaine est une des principales caractéristiques de la production théorique en économie. «Un auteur à prétention théorique doit en premier lieu attester sa maitrise d'une 'littérature' publiée pour l'essentiel en anglais aux États-Unis» (Lebaron 2000 : 131). 
Cela illustre aussi un certain monolithisme de la pensée qui, pour Janos (1986) tient au système universitaire où chaque discipline est constituée de "quelques départements prestigieux dont les professeurs, par leur notoriété et le quadrillage des fondations, revues spécialisées et presses universitaires, contrôlent le débat et la trajectoire professionnelle des membres de la discipline». Les départements d'économie en particulier, comme l'a relevé Galbraith, possèdent une "structure de caste"; au sommet la théorie pure, au plus bas l'économie appliquée (1989 : 131).

Dans cet " univers de l'économiquement correct ", les économistes les plus prestigieux se comportent comme de "nouveaux commissaires idéologiques" (Farnetti-Warde 1997 : 80). Ainsi, Krugman distingue-t-il les vrais économistes qui enseignent dans les grandes universités et publient des articles dans des revues à comité de lecture et les entrepreneurs dont les écrits s'adressent au grand public (1996 : 118-9).

Dans ce contexte, deux langues se sont imposées : l'anglais et les mathématiques. Dans des revues telles que l'American Economic Review, la proportion des pages contenant des mathématiques est passée de moins de $3 \%$ en 1940 à $40 \%$ en 1990 (Beaud 1998: 24). L'accent mis sur la formalisation résulte d'une émulation intellectuelle et du désir de s'intégrer au courant dominant, "mainstream economics ». En effet, progressivement l'économie universitaire a été gagnée puis dominée par ce processus de formalisation, les modèles constituant une part croissante de la matière enseignée en économie (Beaud 1998 : 26). Herbert Simon, Prix Nobel 1978, s'en est lui-même indigné, criant au scandale devant les manuels actuels de microéconomie (1986), tandis que deux rapports réalisés à l'initiative de l'American Economic Association dénoncent cette dérive (Krueger 1991).

Quant à la langue anglaise, en matière économique, c'est elle qui véhicule la nouvelle "pensée unique ». "La science économique est anglo-saxonne» affirmait en 1974 l'économiste Colette Nème. Selon son analyse, «la première condition pour être Prix Nobel de sciences économiques serait donc d'écrire en anglais ou d'être traduit dans cette langue, latin du $20^{\circ}$ siècle » (1974:27).

8 Le recadrage des travaux des économistes universitaires dans le modèle de référence anglo-saxon est constant, à la recherche d'une légitimation intellectuelle. Ainsi, dans l'introduction de l'Analyse macroéconomique de Jean-Olivier Hairault (2000), Jean-Pierre Hénin écrit-il : «[cet ouvrage] constitue aussi un texte de référence empruntant au genre anglo-saxon des handbooks leur ambition de dresser un état des lieux de la discipline ».

Dans le monde professionnel, qu'ils soient universitaires ou non, les économistes sont des producteurs de discours et de rhétorique économique. Ils emploient un langage parfois extrêmement ésotérique, très éloigné du sens commun (Lebaron $2000: 42$ ) alors que l'économiste universitaire britannique Patrick Minford souligne l'intérêt de s'exprimer de façon intelligible : " there is intellectual benefit in having to represent findings in intelligible language $»^{1}$.

En effet, pour un même signifié, diverses appellations se succèdent (variation diachronique) ou coexistent (variation synchronique). "D'un auteur à l'autre, les modifications observées $\mathrm{du}$ vocabulaire correspondent bien souvent à un infléchissement de l'idée centrale visant à l'adapter à une analyse nouvelle " (Lisle 1961 : xxxii). Ainsi Lancaster justifie-t-il le choix du terme characteristics plutôt que celui de utility par la diversité des propriétés des biens déterminant la demande (1971: 
6). Dans ces conditions, un terme n'a de sens qu'au sein de sa théorie ou de son contexte et Samuelson signale ce fait comme un danger :

Especially in the social sciences we must watch out the 'tyranny' of words. The world is complicated enough without introducing further confusions and ambiguities as: 1. Two different names are unknowingly being used for the same thing or: 2. The same word is applied to two different things. (1970:8)

51 Les auteurs de la préface des Essays on Economic Semantics de Fritz Machlup recourent à la même expression :

Professor Machlup has alerted his own students and the profession at large to the tyranny of words. He has been a life-long foe of Mephistopheles, who advised the student in Goethe's Faust to use words to conceal ignorance, to substitute words for what he did not understand. (Machlup $1967: 1$ )

52 Les économistes devraient observer les recommandations de Samuelson contre les pièges de l'originalité créative et pour la recherche d'une écriture directement compréhensible. C'est l'objectif de Mankiw dans la $4^{\mathrm{e}}$ édition de son ouvrage intitulé Macroeconomics:

Together with various editors and students, I have scrutinized each sentence of the book to see whether it can be made clearer. (2000: $\mathrm{xxx})$

My goal in this book is to offer the clearest, most up-to-date, most accessible course in macroeconomics in the fewest words possible. (2000: xxx-xxxi)

... "up-to-date", d'où la nécessité d'introduire les innovations terminologiques, révélant ainsi des variations diachroniques du lexique.

En outre, le transfert des concepts ou de simples dénominations de l'anglais vers le français n'est pas toujours automatique. Certains termes, tels que commodities ont un champ sémantique ne correspondant pas à un terme unique en français (economic goods and services) ; leur adaptation sera la traduction de leur équivalent dans la langue cible ; constrained bliss, traduit par «bien-être maximum sous contrainte » en est un autre exemple.

Pour Mankiw,

An economist must be "mathematician, historian, statesman, philosopher, in some degree [...] as aloof and incorruptible as an artist, yet sometimes as near the earth as a politician. So remarked John Maynard Keynes, the great British economist. (2000 : xxix)

Cette citation explique la diversité des aspects de la langue économique, en particulier la multiplicité des métaphores dont la plus célèbre est sans doute celle de l'invisible hand d'Adam Smith.

Pourquoi l'anglais s'impose-t-il aux économistes universitaires français? Pour Ridler et Pons-Ridler (2000), le choix de la langue reflète le fonctionnement du marché. L'anglais constitue un parfait exemple de l'importance des usagers de la langue seconde pour la vitalité d'une langue. L'importance de l'anglais pour les économistes universitaires est due à l'aura de la recherche anglo-saxonne en économie. La perspective économique donne une dimension particulière au débat autour de la vitalité de la langue. Elle nous force également à examiner la distinction entre la vitalité de la langue et celle de la communauté linguistique elle-même.

En définitive, pour ces économistes universitaires, la maitrise de cette langue anglaise de spécialité exige des compétences dans divers domaines. Pour les préciser, nous recourrons à une typologie des compétences générales présentée par Françoise Demougin (1992) pour l'appliquer aux besoins des économistes universitaires non 
anglophones. Dans le domaine linguistique, ils doivent acquérir la maîtrise d'un système: morphologie, syntaxe, lexique. Dans le domaine discursif, il leur faut maitriser les macro-structures du discours, connaître ses principes, les codes rhétorique et stylistique. Dans le domaine socio-culturel, il faut qu'ils se familiarisent avec les usages particuliers de chaque communauté. Ensuite, dans le domaine référentiel, ils devront maitriser la description du contexte communautaire. Enfin, l'approche diachronique, ou historique, des cultures leur fera remarquer des problématiques semblables ou divergentes, comme dans tout phénomène d'acculturation (Demorgon $2000: 78$ ).

\section{Conclusion}

Après avoir étudié les diverses voies d'émergence du milieu professionnel des économistes universitaires en France, en Angleterre et aux États-Unis, nous avons constaté à quel point ce milieu est caractérisé par le discours anglophone. « Mainstream economics », le courant dominant, s'exprime en langue anglaise. La pré-éminence des revues de langue anglaise en est à la fois une cause et une illustration.

59 Le discours de l'économie a, par ailleurs, tendance à se vulgariser, ce que regrettent certains économistes universitaires, comme Paul Krugman. Alors qu'il était courant, au $20^{\mathrm{e}}$ siècle, de qualifier l'économie de "dismal science ", terme dû au critique anglais Thomas Carlyle dans les années 1850 et communément lié aux prédictions pessimistes de T.R. Malthus, les attitudes ont évolué rapidement. Aujourd'hui, l'économiste Marl Skousen estime que

Like an invading army, the science of Adam Smith is overrunning the whole of social science - law, finance, politics, history, sociology, environmentalism, religion and even sports. Therefore I have dubbed the 21st century economics, the imperial science. (2000)

Certes, en ce début du vingt-et-unième siècle, la science des academic economists est impériale dans son expression anglaise au point de s'imposer aux économistes universitaires $\mathrm{du}$ monde entier. Certains économistes universitaires français considèrent alors de leur devoir d'assurer leurs cours en anglais. Toutefois cela ne saurait se faire sans posséder les compétences linguistiques particulières que nous venons d'évoquer.

\section{BIBLIOGRAPHIE}

Attali, Jacques. 1998. Pour un modèle européen d'enseignement supérieur. Paris : Stock.

Bartoli, Henri. 1998. «La pensée économique au tournant du millénaire ». In L'économie, une science pour l'homme et la société, Mélanges en l'honneur d'Henri Bartoli. Paris : Publications de la Sorbonne, 5-18. 
Beaud, Michel. 1998. «Y a-t-il encore place pour la pensée dans l'univers des économistes universitaires ?». In L'économie, une science pour l'homme et la société, Mélanges en l'honneur d'Henri Bartoli. Paris : Publications de la Sorbonne, 21-36.

Bienaymé, Alain. 1986. L'enseignement supérieur et l'idée d'université. Paris : Economica.

Blaug, Mark. 1999. Who's Who in Economics. Third edition, Cheltenham, UK and Northampton, MA. : Edward Elgar.

Boulding, Kenneth. 1963. « The skills of the economist ». In Anderson, Gitlow \& Diamond (dir), General Economics - A book of Readings. Homewood, Ill : Irwin, Inc.

Coats, A. W. 1993. « The sociology and professionalization of economics ». British and American Essays. Vol. II, Londres : Routledge.

Debreu, Gérard. 2001. «Préface ». Dictionnaire de sciences économiques. Paris : Presses Universitaires de France.

Demorgon, Jacques. 2000. L'interculturation du monde. Paris : Anthropos-Economica.

Demougin, Françoise. 1992. Langue, culture et stéréotypes. Paris : L'Harmattan.

Dudley-Evans, Tony \& Maggie Jo St John. 2001 [1998]. Developments in English for Specific Purposes. Cambridge : Cambridge University Press.

Farnetti, Richard \& Ibrahim Warde. 1997. Le modèle anglo-saxon en question. Paris : Economica.

Fourcade-Gourinchas, Marion. 2002. «Les économistes et leur discours - Traditions nationales et science universelle ». Sciences de la Société 55, février.

Frey, Bruno S. \& Reiner Eichenberger. 2001. « D’où viennent les meilleurs économistes européens ? ». Problèmes économiques 2725, 29 août, 1-12.

Galbraith, Kenneth. 1989. Essays in Persuasion, Cuvres complètes. Vol. IX, Londres : Macmillan.

Hall, P. (dir.). 1989. The Political Power of Economic Ideas. Keynesianism across Nations. Cambridge, MA : Harvard University Press.

Henin, Jean-Pierre. 2000. «Introduction ». In Hairault, Jean-Olivier. Analyse Macroéconomique. Paris : La Découverte.

Hyland, Ken \& Liz Hamp-Lyons. 2002. « EAP: issues and directions ». Journal of English for Academic Purposes 1/1, 1-12.

Janos, A. 1986. Politics and Paradigms. Stanford : Stanford University Press.

Krueger, Ann et al. 1991. « Report of the Commission on graduate education in economics ». Journal of Economic Literature 29 September, 1035-1053.

Krugman, Paul. 1996. Pop Internationalism. Cambridge, MA : The MIT Press.

Lancaster, Kevin. 1971. Consumer Demand, A New Approach. New York : Columbia University Press. Lebaron, Frédéric. 2000. La Croyance économique - Les économistes entre science et politique. Paris : Seuil.

Lebaron, Frédéric. 2002. "Le 'Nobel' d'économie. Une politique ». Actes de la Recherche en Sciences Sociales 141-142, mars, 62-69.

Lisle, Edmond. 1961. L'anglais économique. Paris : Éditions Cujas.

Machlup, Fritz. 1967. Economic Semantics. New York: W. W. Norton. 
Mankiw, Gregory. 2000. Macroeconomics. $4^{\mathrm{e}}$ édition. New York : Worth Publishers.

Moore, William J., Robert J. Newman \& M. Dek Terrell. 2002. « Academic economists' pay and productivity - A tale of two countries ». Baton Rouge, LA, Louisiana State University, Department of Economics, novembre 2002 <http://www.bus.lsu.edu/economics/papers/pap02_16.pdf>.

Nème, Colette. 1974. « Les Prix Nobel de l'économie ». Problèmes économiques 1372, 15 mai, 27.

Ridler, Neil \& Suzanne Pons-Ridler. 2002. «L'influence des usagers de la langue seconde », novembre 2002 <http://www.pch.gc.ca/progs/lo-ol/perspectives/francais/assimil2/01d.html>.

Samuelson, Paul. 1970. Economics. 8th ed. Cambridge, MA : McGraw-Hill.

Simon, Herbert. 1986. «The failure of armchair economics ». Challenge 29/5, 18-25.

Skousen, Mark. 2001. The Making of Modern Economics. Boston, MA : M.E. Sharpe.

Swales, John. 1990. Genre Analysis: English in Academic Settings and Research. Cambridge : Cambridge University Press.

Touraine, Alain. 1972. Université et société aux États-Unis. Paris : Éditions du Seuil.

Trudgill, Peter. 1995 [1974]. Sociolinguistics, an introduction to language and society, édition revue. Londres : Penguin Books.

Wright, V. \& F. Marx. 1973. Les universités britanniques. Paris : Presses Universitaires de France.

\section{ANNEXES}

Boulding, Kenneth. 1963. "The Skills of the Economist "

Before we examine the skill of the economist we should ask, "Who are the economists?" This is an embarrassing question. There are no recognized tests by which economists can be distinguished from those who may claim but who do not deserve the name. We have no professional qualifying examination as do the lawyers, the doctors, the accountants, and in some places, I understand, the beauticians. I know of no one who has been prevented from joining the American Economic Association or any other economic association for reasons of professional incompetence. We have no priesthood guarding the sacred fire handed down from Adam Smith. We do not even follow the practice of so many professions in improving their economic status by raising barriers to entrance. We may understand monopoly but we certainly do not practice it. Indeed, we are one of the few professions which deliberately, it would seem, attempts to undermine its economic status by actually encouraging students to enter it and by refusing to impose any professional standards.

Some unkind souls may want to deny us the status of a profession altogether. Moreover, it is a common belief that where two or three economists are gathered together there are always three or four varieties of economics present. We are popularly supposed to be divided by schools and racked by dissent, speaking with no common voice and being therefore quite unworthy of the name of science.

If these things be true, it is an act of daring to claim there is a skill of the economist. Nevertheless, in spite of the divisions among us, and in spite of many signs of scientific immaturity, my experience with non economists convinces me there is something, 
however humble, which can properly be called skill among those who recognize themselves as economists.

\section{NOTES}

1. Patrick Minford, novembre 2002 < http://www.esrc.ac.uk/esrccontent/PublicationsList/ 4books/hero9.html>.

\section{RÉSUMÉS}

Cet article porte sur la communauté des économistes universitaires telle qu'elle apparaît en France, en Angleterre et aux États-Unis. Après avoir exposé ses caractéristiques institutionnelles et culturelles et les indices de professionnalisation dans chacun de ces pays, il met en évidence dans son discours l'existence de deux éléments prépondérants dans ces pays comme dans le monde entier: la langue anglaise et les mathématiques. Pour ce qui concerne la langue, cet article s'inscrit à la fois dans le domaine de l'anglais de spécialité puisqu'il s'agit d'anglais économique et dans celui de l'anglais académique puisqu'il observe le discours d'une communauté universitaire.

This paper focuses on the emergence of the community of academic economists in France, England and the United States. After delineating its institutional and cultural specificities and signs of professionalization in each of these countries, it stresses the major role played in its discourse by the English language and mathematics, in these countries and all over the world. As far as language is concerned, this paper falls both within the field of English for Specific Purposes, as it deals with English for economics, and within the field of English for Academic Purposes, since it studies the discourse of an academic community.

\section{INDEX}

Mots-clés : communauté linguistique, discours, économiste universitaire, professionnalisation

Keywords : discourse, economic academic, linguistic community, professionalization

\section{AUTEUR}

\section{JACQUELINE PERCEBOIS}

Jacqueline Percebois est professeur ( $11^{\mathrm{e}}$ section du CNU) à l'Université Aix-Marseille 1 où elle est responsable du Master LEA Métiers de la Traduction et directrice adjointe du Département d'Études du monde anglophone. Elle est membre de l'EA 2025 (Bordeaux 2). Ses travaux de recherche portent sur le discours et la terminologie comparée anglais-français en contextes économiques. Ils ont donné lieu à la publication d'articles et de trois ouvrages mettant en parallèle les terminologies anglaise et française des théories microéconomique et 
macroéconomique ainsi que des théories, politiques, accords et institutions du commerce international. jacqueline.percebois@univ-provence.fr 